\title{
$8-2020$
}

\section{Direct true lumen versus conventional cannulation for acute type- A aortic dissection}

Asra Wahid

Syed Shahabuddin

Muhammad Muneer Amanullah

Shiraz Hashmi

Shahid Ahmed Sami

Follow this and additional works at: https://ecommons.aku.edu/pakistan_fhs_mc_mc

Part of the Cardiology Commons, Cardiovascular Diseases Commons, and the Surgery Commons 


\section{STUDENTS' CORNER SHORT COMMUNICATION}

\section{Direct true lumen versus conventional cannulation for acute type-A aortic dissection}

Asra Wahid1, Syed Shahabuddin², Muhammad Muneer Amanullah³, Shiraz Hashmi ${ }^{4}$, Shahid Sami ${ }^{5}$

\begin{abstract}
Acute type-A aortic dissection is a surgical emergency and has a high rate of short-term mortality. Aortic dissection is highly under-reported in Pakistan. With the technological developments in its management, arterial cannulation technique of direct true lumen cannulation has emerged with improved outcomes. We aimed to compare the mortality and morbidity outcomes between direct true lumen and conventional cannulation techniques for arterial access in patients with acute type$A$ aortic dissection under a single-centre retrospective review from 2007 to 2017. Mean age of the participants was $43.3 \pm 11.6$ vs $45 \pm 12.4$ years with males being dominant in both groups. Frequency of overall morbidity was high in conventional cannulation group (Group-B), though it did not attain statistical significance, ( $p>0.999)$. Mortality rate was also high in Group-B (10\% vs $30 \%),(p=0.582)$. Direct true lumen cannulation is an equally reliable option for establishing cardiopulmonary bypass due to reduced mortality and morbidity and may be given preference when dissection is extending into femoral and innominate arteries.
\end{abstract}

Keywords: Acute aortic dissection, type-A dissection, cardiopulmonary bypass, cannulation.

\section{https://doi.org/10.5455/JPMA.14771}

\section{Introduction}

Acute type-A Aortic dissection (AAAD), a challenging clinical and surgical emergency, is associated with a high rate of short-term mortality of around (8-34\%). ${ }^{1}$ Main challenges associated with this type of procedure include establishing adequate extracorporeal circulation, resecting the torn intima, and protecting vital organs, primarily the brain from ischaemia. ${ }^{2}$

Access to arterial flow can be multiple and are debatable. The conventional method of retrograde perfusion

1 Final Year Medical Student, Aga Khan University, Karachi, Pakistan; 2,4,5 Department of Surgery, Aga Khan University, Karachi, Pakistan; ${ }^{3}$ Department of Surgery, National Institute of Cardiovascular Diseases, Karachi, Pakistan. Correspondence: Syed Shahabuddin. e-mail: syed.shahab@aku.edu technique using femoral artery cannulation increases the chances of false lumen perfusion, organ malperfusion and risk of stroke. ${ }^{3}$ Antegrade flow established via axillary artery is time consuming, may cause arterial injury and insufficient flow problems; while cannulating the aorta directly, poses the risk of cannulating the false lumen.4,5 A modified technique of direct true lumen cannulation (TLC) can solve these problems. Jakob et al and Conzelmann et al proposed that, under direct vision, cannulation of the true lumen of the ascending aorta could be a safe and rapid method of antegrade arterial perfusion in AAAD with minimal mortality and neurological complications. ${ }^{6,7}$ Yamamoto and Kitamura et al's comparison between direct TLC and other cannulation strategies reported no cannulation complications along with reasonable mortality and stroke rates. ${ }^{8,9}$ Along with the scarcity of literature on aortic dissection in Pakistan, this new technique has only been recently introduced in the country at Aga Khan University Hospital. No study is available in Pakistan on this subject, while only sparse comparisons have been made worldwide. 8,9 Therefore, we aimed to compare demographic, clinical characteristics, peri-operative and the outcome variables of a newly established direct TLC technique with conventional techniques of cannulation during type-A dissection surgeries at our centre in Pakistan.

\section{Methods}

All patients operated for AAAD from 2007 to December 2017 at the Aga Khan University Hospital, Karachi were included in the descriptive, retrospective analytical chart review. Patients were categorised into two groups based on arterial cannulation strategy used (i.e. direct TLC and conventional cannulation strategies) during cardiopulmonary bypass (CPB). Only 10 TLC eligible cases were identified to be included in this study after excluding five cases with incomplete data in the charts. Their age and gender matched controls (conventional cannulation) were selected in the ratio of 1:1. After ERC approval (5276-SurERC-18), medical charts were retrieved and reviewed. The 
standard operating protocol was used for femoral, axillary and central aortic cannulation. Direct TLC was performed as described by Jakob6 and Conzelmann7 along with our modifications. ${ }^{10}$ All statistical analyses were performed using IBM SPSS Statistics version 21.0. Normality of all continuous variables was determined. Student t-test for normally distributed variables or Mann Whitney- $U$ test for skewed data was applied to assess group differences. Likewise, Chi-squared or Fisher's exact test was applied for categorical data. A p-value $<0.05$ was considered statistically significant.

\section{Results}

Our study includeda 20 patients. Direct TLC was performed in 10 patients (Group-A) and other cannulation strategies were performed in the other 10 patients (Group-B). Table 1 demonstrates the comparison of demographics and preoperative characteristics of both groups. Age: $43.3 \pm 11.6$ vs $45.0 \pm 12.4$ years, $(p=0.755)$, proportion of male gender: $80 \%$ vs 70\%, ( $p>0.999)$ and BMI: $26.2 \pm 6.6$ vs $26.3 \pm 5.6 \mathrm{~kg} / \mathrm{m} 2$, $(p=0.959)$ were comparable between the groups. Main diagnostic tools used were echocardiography and CT Angiogram. All patients were found to have aortic valve regurgitation.

Operative variables are described in Table 2. Thirteen (65\%) patients had aortic root replacement with a composite graft and a mechanical valve placement. Six (30\%) patients had valve-sparing aortic root replacement. Only one $(5 \%)$ patient underwent a hemi-arch replacement along with aortic valve resuspension. All 10 patients in Group-A underwent direct TLC. Of the 10 patients in Group-B, CPB was established via retrograde perfusion using femoral artery cannulation in six (60\%) patients, and via antegrade perfusion using axillary artery cannulation and central aortic cannulation in two patients each (40\%). Amongst arterial injury complications in Group-B, one patient had bleeding via the femoral artery and received a femoral artery patch repair, while the other patient had a failed axillary artery cannulation while cannulating a haemodynamically unstable patient, therefore, the femoral artery was cannulated instead.

Outcome measures are depicted in Table 3. One patient in Group-A died during hospital stay due to multiple organ failure, sepsis and cardiogenic shock. In Group-B out of three, two patients died during hospital stay due to cardiogenic shock and one died within 30 days of the index procedure due to excessive bleeding leading to
Table-1: Comparison of Demographic and Clinical characteristics in True Lumen Cannulation Vs Conventional Cannulation.

\begin{tabular}{lccc}
\hline Variables & \#Group-A, $\mathbf{n}=\mathbf{1 0}$ & tGroup-B, $\mathbf{n}=\mathbf{1 0}$ & $\boldsymbol{p}$-value \\
\hline Mean Age (years) & $43.3 \pm 11.6$ & $45.0 \pm 12.4$ & 0.755 \\
Gender (male) $\mathrm{n}(\%)$ & $8(80.0)$ & $7(70.0)$ & $>0.999$ \\
Mean BMI $(\mathrm{Kg} / \mathrm{m} 2)$ & $26.2 \pm 6.6$ & $26.3 \pm 5.6$ & 0.959 \\
Mean SBP (mmHg) & $112.3 \pm 28.3$ & $107.7 \pm 27.3$ & 0.715 \\
Pre-Operative Variables & & & \\
Hx of HTN n(\%) & $8(80.0)$ & $8(80.0)$ & $>0.999$ \\
Hx of CHF n(\%) & Nil & $3(30.0)$ & 0.211 \\
Hx of AKI n(\%) & $1(10.0)$ & $3(30.0)$ & 0.582 \\
Hx of MI n(\%) & $1(10.0)$ & $1(10.0)$ & $>0.999$ \\
Mean Pre-op Echocardiography LVEF\% & $46.1 \pm 16.1$ & $51.4 \pm 12.8$ & 0.488 \\
Pericardial effusion n(\%) & $3(30.0)$ & $5(50.0)$ & 0.650 \\
Pericardial Tamponade n(\%) & $1(10.0)$ & $3(30.0)$ & 0.582 \\
Pre-op Shock (SBP<80mmHg) $\mathrm{n}(\%)$ & $2(20.0)$ & $5(50.0)$ & 0.350 \\
\hline
\end{tabular}

‡True Lumen Cannulation; †Conventional Cannulation; ${ }^{*} \pm$ SD: Standard Deviation BMI: body mass index; SBP: Systolic Blood Pressure; HTN: Hypertension; CHF: Congestive Heart Failure; AKI: Acute Kidney Injury; MI: Myocardial Infarction; LVEF: Left Ventricular Ejection Fraction

Table-2: Comparison of Peri-operative variables in True Lumen cannulation vs Conventional Cannulation.

\begin{tabular}{|c|c|c|c|}
\hline Variables & $\begin{array}{c}\begin{array}{c}\text { \#Goup-A } \\
n=10\end{array} \\
\end{array}$ & $\begin{array}{c}\text { tGroup-B } \\
n=10\end{array}$ & $p$-value \\
\hline Mean Total procedure time (min) & $424( \pm 58.9)$ & $457( \pm 101.3)$ & 0.397 \\
\hline Mean CBP Time (min) & $237.7( \pm 35.2)$ & $233.6( \pm 50.4)$ & 0.835 \\
\hline Mean Cross Clamp Time (min) & $142.6( \pm 61.8)$ & $152( \pm 51.5)$ & 0.706 \\
\hline Mean Circulatory Arrest Time (min) & $35.7( \pm 6.1)$ & $30.7( \pm 8.7)$ & 0.185 \\
\hline Mean Hypothermic Circulatory Arrest $\left({ }^{\circ} \mathrm{C}\right)$ & $17.15( \pm 0.9)$ & $23.0( \pm 5.5)$ & 0.005 \\
\hline Antegrade Cerebral Perfusion n(\%) & $10(100.0)$ & $4(40.0)$ & 0.011 \\
\hline Mean Hospital Length of Stay (days) & $7.95( \pm 4.3)$ & $11.0( \pm 10.2)$ & 0.359 \\
\hline ICU Stay (days) median (IQR) & $3.0(1.9,6.0)$ & $3.0(2.0,8.5)$ & 0.661 \\
\hline Mean Intubation Time (hrs) & $42.7( \pm 34.3)$ & $55.8( \pm 48.4)$ & 0.459 \\
\hline Cannulation Complications n(\%) & Nil & $2(20.0)$ & 0.474 \\
\hline
\end{tabular}

LCOS: Low Cardiac Output Syndrome; MI: Myocardial Infarction; Afib: Atrial fibrillation; Median (Interquartile range), Mann Whitney U test applied.

Table-3: Comparison of Outcomes in True Lumen cannulation vs Conventional Cannulation.

\begin{tabular}{|c|c|c|c|}
\hline Variables & $\begin{array}{c}\neq \text { Group }-A \\
n=10\end{array}$ & $\begin{array}{c}\text { tGroup-B } \\
n=10\end{array}$ & $p$-value \\
\hline Mortality n(\%) & $1(10.0)$ & $3(30.0)$ & 0.582 \\
\hline Overall Morbidity§n(\%) [A(n=9), $B(n=7)]$ & $4(44.4)$ & $4(57.1)$ & $>0.999$ \\
\hline Multiple Organ Failure n(\%) & Nil & $3(42.9)$ & 0.063 \\
\hline Respiratory Failure n(\%) & $1(11.1)$ & $2(28.6)$ & 0.550 \\
\hline Acute Renal failure $\mathrm{n}(\%)$ & $2(22.2)$ & $3(42.9)$ & 0.596 \\
\hline Heart Failure/LCOS n(\%) & $1(11.1)$ & $2(28.6)$ & 0.550 \\
\hline Sepsis n(\%) & Nil & $1(14.3)$ & 0.438 \\
\hline Acute $M \ln (\%)$ & $1(11.1)$ & Nil & $>0.999$ \\
\hline Arrhythmia/Afibn(\%) & Nil & $1(14.3)$ & 0.438 \\
\hline Follow Up Time (weeks) median (IQR) & $44.0(1.5,126)$ & $65.0(8.0,148)$ & 0.681 \\
\hline
\end{tabular}

Vol. 70, No. 8, August 2020 
cardiogenic shock.

No significant difference was seen in the post-operative complications amongst the surviving patients of the two groups. There was no neurological injury reported in any group. Overall median follow-up time in both the groups was 46, IQR (5.0-131) weeks, Group-A: 44.0, IQR (1.5-126) vs. Group-B: 65.0, IQR (8.0-148) weeks respectively. Overall follow up rate was $75 \%$ and the functional class improved in $91.6 \%(11 / 12)$ from III-IV to I-II. No mortality or reintervention were reported amongst these patients up till the last follow-up.

\section{Discussion}

AAAD has an incidence of around 30 cases per million individuals per year ${ }^{11}$ and its management requires an emergent open-heart surgery despite the increased risk of postoperative mortality and morbidity. ${ }^{12}$ In Pakistan, there is a huge scarcity of literature on aortic disease. From 1988-2015, only 19 cases of aortic dissection have been documented in the country with a mortality rate of $47.3 \%$ (9/19).13,14 In our study, we found in-hospital mortality rate of $20 \%$ amongst AAAD cases which is comparable to internationally published rates ranging from 21-33\%. ${ }^{15}$

FAC is the traditional, fastest and easiest accessible site, therefore, its use is preferred in haemodynamically unstable patients instead of axillary approach which may occasionally be too time-consuming. ${ }^{16,17}$ The use of FAC, primarily due to a retrograde flow in the aorta, has the maximum rate of mortality, false lumen perfusion, limb ischaemia, coronary and cerebral embolisation and organ malperfusion. Axillary artery cannulation technique has a theoretical advantage in providing an antegrade flow during the cooling period. 16,18 However, cannulation problems of the axillary artery are reported to be as high as $10 \% .4,5$

In our study, one patient had difficulty being cannulated via axillary artery due to haemodynamic instability and therefore, the femoral artery was used instead.

Since both peripheral cannulation techniques have certain advantages and disadvantages for AAAD patients, Jakob et al directly cannulated the true lumen during $A A A D$ surgery. ${ }^{6}$ Jakob et al and Conzelmann et al., each, in their case series, reported a $0 \%$ mortality rate.6,7 Yamamoto et al reported an $8 \%$ mortality rate amongst the TLC group compared to an $11.4 \%$ mortality rate in the other cannulation group of antegrade flow. 9 In our study, however, one patient died out of ten amongst Group-A nevertheless, the death was unrelated to cannulation technique per se. Recently, Kitamura et al conducted a similar comparison and reported reasonable stroke and mortality rates and no cannulation complications. ${ }^{8}$ Group$A$ and $B$ specific mortality was estimated to be $10 \%$ vs $30 \%$ and morbidity was ( $44.4 \%$ vs $57.1 \%$ ), however, no statistical group difference was detected.

Neurological complication is reported to be $21.1-25.3 \%$ amongst TLC patients, 6,7 while amongst direct aortic cannulation it is reported to be $3.8-21.4 \%$, with femoral artery cannulation $3.0-26.4 \%$ and with axillary artery cannulation (1.8-14.3\%) respectively.3,19 We had no neurological complications and cannot comment on it. Kitamura et al reported an $8 \%$ cannulation complication rate in the other groups with no cannulation complications in the TLC group. ${ }^{8}$ Likewise, we had only two patients in the other group with arterial injury complications. The results of our study showed that direct TLC has minimal complications; it spares peripheral arteries from any injuries and consumes less time in establishing CPB.

\section{Conclusion}

Direct TLC ensures antegrade perfusion to the brain and other organs through the true lumen and may improve the surgical outcomes. With experience this option of arterial access is as good as conventional techniques, and in some cases where dissection is extending into femoral and innominate artery, it may be the preferred technique of cannulation.

Disclaimer: This research has been presented at the 27th European Cardiology conference in Rome Italy, in October 2018.

Conflict of Interest: None. Funding Disclosure: None.

\section{References}

1. Antonio Lio, Francesca Nicolò, Emanuele Bovio, Andrea Serrao, Jacob Zeitani, Antonio Scafuri, et al. Total Arch versus Hemiarch Replacement for Type A Acute Aortic Dissection: A Single-Center Experience. Tex Heart Inst J. 2016; 43: 488-95.

2. Po-Shun Hsu, Chien-Sung Tsai, Yi-Ting Tsai, Chih-Yuan Lin, ChungYi Lee, Hong-Yan Ke, et al. Subclavian artery cannulation provides better myocardial protection in conventional repair of acute type A aortic dissection: experience from a single medical centre in Taiwan: cardiovascular topics. Cardiovasc J Afr. 2016; 27: 143-6.

3. Tiwari KK, Murzi M, Bevilacqua S, Glauber M. Which cannulation (ascending aortic cannulation or peripheral arterial cannulation) is better for acute type A aortic dissection surgery? Interact Cardiovasc Thorac Surg. 2010; 10:797-802. 
4. Sabik JF, Nemeh H, Lytle BW, Blackstone EH, Gillinov AM, Rajeswaran $\mathrm{J}$, et al. Cannulation of the axillary artery with a side graft reduces morbidity. Ann Thorac Surg. 2004; 77:1315-20.

5. Parachuri VR, Subramanian A. True Lumen Perfusion Technique for Extensive Aortic Dissections Involving the Neck and Femoral Vessels. Ann Thorac Surg. 2017; 104:e295-7.

6. Jakob H, Tsagakis K, Szabo A, Wiese I, Thielmann M, Herold U. Rapid and safe direct cannulation of the true lumen of the ascending aorta in acute type A aortic dissection. J Thorac Cardiovasc Surg. 2007; 134: 244-5.

7. Conzelmann LO, Kayhan N, Mehlhorn U, Weigang E, Dahm M, Vahl CF. Reevaluation of direct true lumen cannulation in surgery for acute type A aortic dissection. Ann Thorac Surg. 2009; 87:1 182-6.

8. Kitamura T, Torii S, Kobayashi K, Tanaka Y, Sasahara A, Ohtomo Y et al. Samurai cannulation (direct true-lumen cannulation) for acute Stanford Type A aortic dissection. Eur J Cardiothorac Surg. 2018; 54:498-503.

9. Yamamoto N, Nie M, Hari Y, Tanaka Y, Ohara K, Miyaji K. A selection of cases of direct cannulation in surgery for type A dissection. Asian Cardiovascular and Thoracic Annals. 2014; 22: 284-7.

10. Sami SA, Shahabuddin S, Khan FW. A selection of cases of direct cannulation in surgery for type A dissection. Asian Cardiovasc Thorac Ann. 2014; 22:887.

11. Coady MA, Rizzo JA, Goldstein LJ, Elefteriades JA. Natural history, pathogenesis, and etiology of thoracic aortic aneurysms and dissections. Cardiol Clin. 1999; 17:615-35.
12. Criado FJ. Aortic Dissection: A 250-Year Perspective. Tex Heart Inst J. 2011 ; 38:694-700.

13. Khan NKU, Razzak JA, Sharif H, Qazi SH. Non-Traumatic Aortic Emergencies-Experience from a Tertiary Care Centre in. J Pak Med Assoc. 2005; 55:6-10.

14. Muhammad ZU, Niaz A, Muhammad KA, Aurangzeb D, Azam ST, Fazl e Rabi, et al. ACUTE TYPE AAORTIC DISSECTION: A PATHOLOGY UNDER DIAGNOSED. J Postgrad Med Inst. 2011; 21.

15. Pape LA, Awais M, Woznicki EM, Suzuki T, Trimarchi S, Evangelista A, et al. Presentation, diagnosis, and outcomes of acute aortic dissection: 17-year trends from the International Registry of Acute Aortic Dissection. J Am Coll Cardiol. 2015; 66:350-8.

16. Abe T, Usui A. The cannulation strategy in surgery for acute type A dissection. Gen Thorac Cardiovasc Surg. 2017; 65:1-9.

17. Klotz S, Bucsky BS, Richardt D, Petersen M, Sievers HH. Is the outcome in acute aortic dissection type $A$ influenced by of femoral versus central cannulation? Ann Cardiothorac Surg. 2016; 5:310-6.

18. Shimokawa T, Takanashi S, Ozawa N, Itoh T. Management of intraoperative malperfusion syndrome using femoral artery cannulation for repair of acute type A aortic dissection. Ann Thorac Surg. 2008; 85:1619-24.

19. Ren Z, Wang Z, Hu R, Wu H, Deng H, Zhou Z, et al. Which cannulation (axillary cannulation or femoral cannulation) is better for acute type A aortic dissection repair? A meta-analysis of nine clinical studies. Eur J Cardiothorac Surg. 2015; 47:408-15. 